\title{
Major Technology and Business Trends Empowering Stem Cell Reprogramming
}

\section{Cauwenberghe $\mathrm{CV}^{*}$}

Favaloro University, Argentina

*Corresponding author: Cecilia Van Cauwenberghe, Associate Fellow and Senior Industry Analyst in the TechVision Group at Frost \& Sullivan, Favaloro University, Argentina, Tel: +541147771333; Email: cecilia.vancauwenberghe@frost.com

\section{Mini Review \\ Volume 3 Issue 2}

Received Date: September 25, 2019

Published Date: October 17, 2019

DOI: $10.23880 /$ jes-16000129

\section{Abstract}

The advent of stem cells reprogramming commenced with the discovery of the differentiation factors that allow the creation of human iPSCs (hiPSCs). Today, researchers all around the world are strongly committed to develop novel methods to design and build human stem cells in order to meet the increasing demand for more efficient production systems and manufacturing methods of stem cells for potential investigation into disease management. This trend is emphatically envisaged to accelerate the development of both regenerative medicine and drug discovery and development.

Keywords: Regenerative Medicine; Stem Cells; Gene Editing; Cell Reprogramming; Cell Therapy; Gene Therapy; Three Dimensional Bioprinting; Nanotechnology; Induced Pluripotent Stem Cells; Tissue Engineering

Abbreviations: CAGR: Compound Annual Growth Rate; ASCs: Adult stem cells; NSCs: Neuronal Stem Cells (NSCs), HSCs: Hematopoietic Stem Cells; MSCS: Mesenchymal Stem Cells; UCSCs: Umbilical Cord Stem Cells; HESCS: Human Embryonic Stem Cells; NAM: Nicotinamide; NK: Natural Killer; FDA: Food and Drug Administration.

\section{Trends Energizing the Cell Reprogramming Sector}

\section{Roadmap Tapping into Technology Synergy}

Stem cell reprogramming concerns the conversion of a somatic cell to an induced pluripotent stem cell (iPSC) in a previous stage to its differentiation into one of many diverse cell types. During the past five years, reprogramming optimization has gained increasing attention. Although one of the most promising technologies ever seen in medical biology, stem cells reprogramming methodology is still challenging. Indeed, most techniques use vectors to integrate DNA into the cell genome. As a consequence, potential issues may appear into scene, including insertional mutagenesis and residual expression of reprogramming factors in cellular progeny. Moreover, integrating vectors are not able to preserve the original genomic integrity of the somatic cell, hence making the therapeutic potential of iPSC limited. Recent attempts are focused on using non-integrating vectors, leveraging many different technology strategies [1].

Although, stem cells reprogramming encompasses radical restructuring of both the metabolism and the epigenome, iPSCs retain transcriptional, epigenetic, and metabolic memories from their originator somatic cells of origin. The correlation between the microenvironment and regulation of the epigenome constitute a critical aspect to take care during the process. Otherwise, 


\section{Journal of Embryology \& Stem Cell Research}

reprogrammed cells may acquire aberrant characteristics distinct from either other pluripotent cells or parental cells revealing incomplete reprogramming. On that note, a key role is played by nutrient availability during the retention of somatic cell memory by iPSCs, hence reflecting satisfactory reprogramming efficiency, epigenetic regulation and cell fate [2]. An in-depth understanding of the current advances in reprogramming pluripotency will inspire scientists to devise more efficient methods that will lead to breakthrough clinical [3]. In fact, remarkable efforts have been made to identify the potential miRNA-mRNA interactions aiming for a better perception of their role in regulating the cellular transitions in different cell lines and their reprogrammed iPSCs through exhaustive genome-wide miRNA and mRNA expression analyses [4]. Similar endeavors are focused on achieving a more profound knowledge on the metabolic features of pluripotent stem cells and the mechanisms of the metabolic shift during reprogramming from somatic cells to iPSCs, in which the metabolism switches from oxidative phosphorylation to glycolysis [5].
Novel strategies are being developed in order to improve stem cells reprogramming by overcoming principal barriers, such as the reprogramming inhibition. The use of non-integrative delivery methods, overexpression of enhancing genes and the use of certain small molecules are some instances of enhanced stem cells reprogramming.

\section{Therapeutic Companies are Looking for Ideally Suited Series of Products that Target Specific Market Needs}

Stem cells reprogramming is highly tied to the remarkable growth of the induced pluripotent stem cells market segment, which is presenting the most promising growth trend. According to Frost \& Sullivan's market dynamics analysis for the period 2018-2023 [6], the global cell reprogramming market was valued in $\$ 5.67$ billion in 2018 and it is expected to reach $\$ 9.05$ billion in 2023 , growing at a compound annual growth rate (CAGR) of $9.8 \%$, as it is exhibited in the Figures $1 \& 2$.

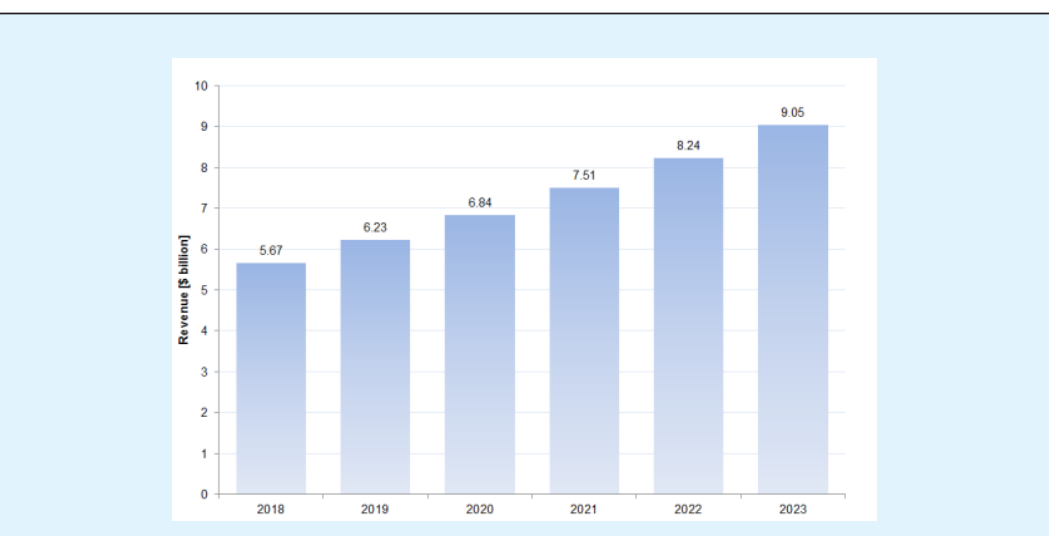

Figure 1: Stem Cells, Overall Market Forecast, Global, 2019-2023.

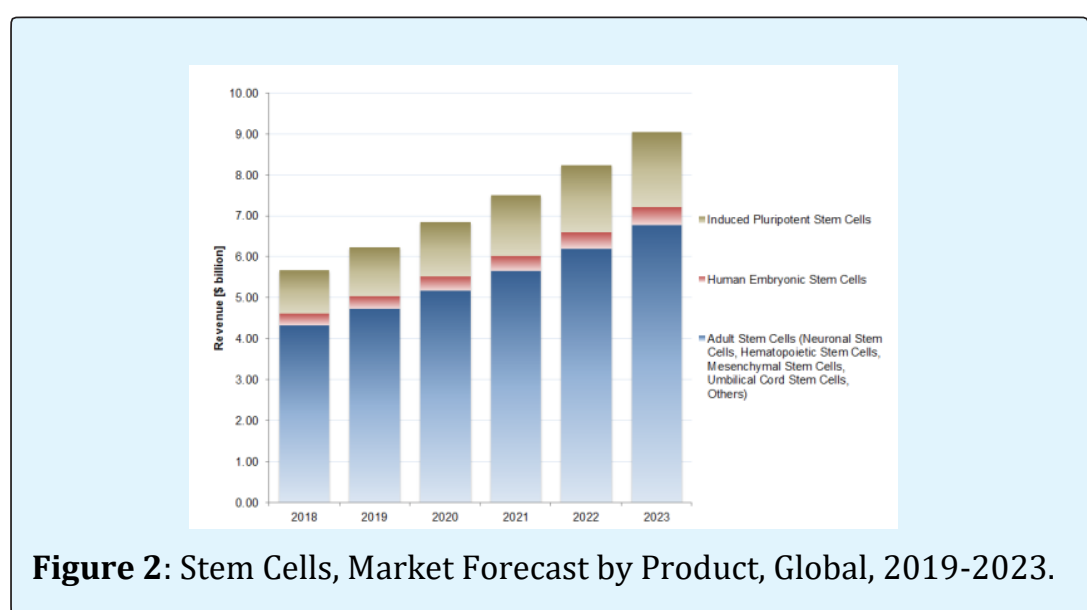




\section{Journal of Embryology \& Stem Cell Research}

Adult stem cells (ASCs), including neuronal stem cells (NSCs), hematopoietic stem cells (HSCs), mesenchymal stem cells (MSCs), and umbilical cord stem cells (UCSCs), are forecasted to continue growing at a CAGR of $9.4 \%$. Human embryonic stem cells (hESCs) present the lowest growth trend with a CAGR of $8.7 \%$, whereas iPSCs are expected to grow at a CAGR of $11.2 \%$, showing the greatest growth trend in the stem cells market. This fact significantly encourages the advent of technologies in stem cells reprogramming.

Regarding applications, regenerative medicine advances in various therapeutic research areas, such as neurology, orthopedics, oncology, hematology, diabetes, liver disorder, kidney disease, cardiovascular and myocardial infraction, injuries, are predicted to grow at a CAGR of $9.7 \%$ during the forecasted period. Drug discovery and development applications present higher growth projection with a CAGR of $9.9 \%$.

In terms of sources, considered most effective, but complex therapy, autologous sources are expected to grow at a CAGR of $9.6 \%$ during the forecast period. Allogeneic sources show the greatest growth trend, with a CAGR of $10.0 \%$. Syngeneic sources are also promising, exhibiting a CAGR of $9.5 \%$.
Concerning product developers, therapeutic companies will exhibit a major growth trend during 20182023 with a remarkable CAGR of $12.0 \%$. Cell and tissue banks have gained considerable attention during the past five years, and are presently projected to grow at a CAGR of $9.0 \%$. Similarly, tools and reagent companies are dramatically proactive in the market and are predicted to reach a CAGR of $9.9 \%$ for the period under study.

Comparably, service companies are constantly rebuilding themselves to offer the best suited stem cells based services to their customers, expecting to achieve a CAGR of $9.8 \%$ during the next five years.

Cell acquisition from bone marrow harvest, umbilical blood cord, and apheresis, is projected to reach a CAGR of $8.5 \%$. Cell production via therapeutic cloning, in-vitro fertilization, cell culture, and isolation techniques, is expected to grow at a CAGR of $10.0 \%$ during the period under study. Cryopreservation technologies present an increasing growth rate of $10.6 \%$.

Most notable, expansion and sub-culture technologies, especially involving three-dimensional cell culture, are expected to grow at a higher CAGR, achieving 11.3\% growth rate during the next five years, as it is depicted in the Figure 3.

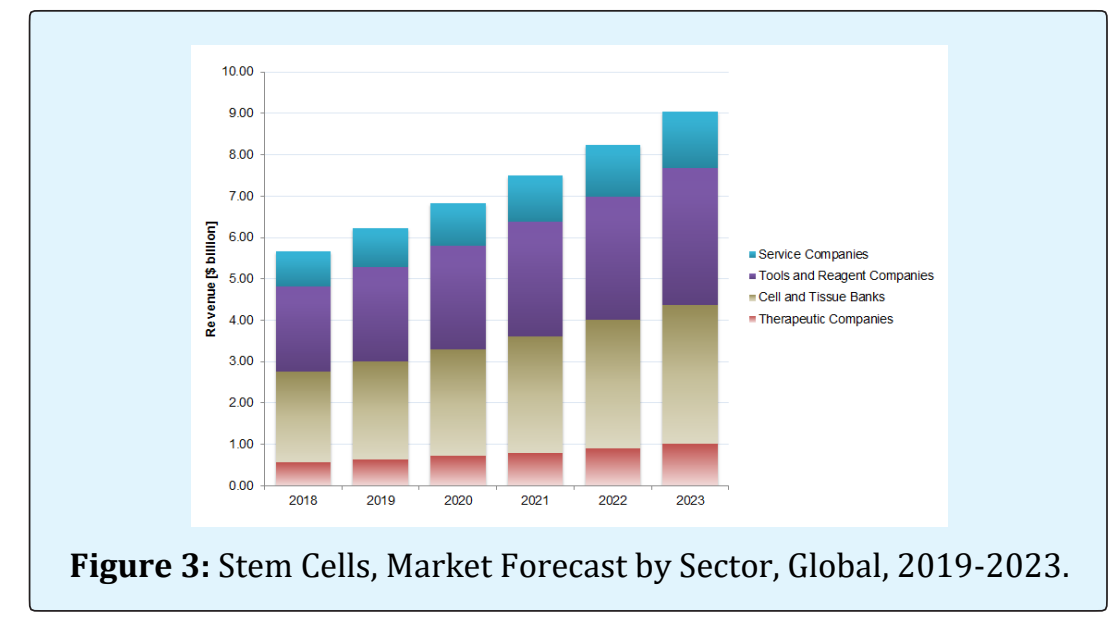

According to Frost \& Sullivan's analysis, North America is still leading the market with $43 \%$ market share. The region is expected to grow at a CAGR of $9.3 \%$. Representing $30.7 \%$ of market share, Europe is projected to achieve a CAGR of $9.5 \%$, similar to North America. Although representing just $22.2 \%$ of market share today, the Asia Pacific region is expected to lead the growth rate with a CAGR of $11.6 \%$ during the next five years.

\section{Major Focus on Stem Cells Reprogramming}

\section{Most Prospective Technology Trends Accelerating Clinical Interventions of Stem Cells Reprogramming}

The field of regenerative medicine deals with serious ethical concerns around the use of embryonic materials. 


\section{Journal of Embryology \& Stem Cell Research}

Nevertheless, the advent of reprogramming technologies has introduced a paradigm shift in therapeutics development. Novel cell reprogramming techniques, such as direct reprogramming, allow a somatic cell to switch from one type to another in vitro or in vivo, by using the best suited transcription factors. Direct cell reprogramming allows generating autologous neural precursor cells that can be used as the starting material for any type of patient's cell to fight diseases associated with central nervous systems. In the cell reprogramming space interesting technology platforms to watch are being highlighted due to both its innovative profile and its capability to enter the market. Fortuna Fix pioneers reprogramming of stem cells by using autologous neural precursor cells produced by direct reprogramming (drNPCs). Fortuna Fix' patented technology portfolio also comprises end-to-end bio manufacturing process automation aiming to accelerate scale-up, production, and commercialization. Fortuna Fix' proprietary technology would accelerate the development of efficient and definitive clinical therapeutics to treat many neurological diseases. Fortuna Fix lead therapy consists of autologous A9 dopaminergic neuronal precursor cells (drNPC-A9) for Parkinson's disease and oligo-dendroglia-biased drNPCs for spinal cord injury. Chromatin remodeling techniques are used to keep the master and secondary genes expression stable, while locking into the particular neural pluripotent epigenetic state. Master and regulatory genes control the expression of a number of secondary genes, which directly reprogram the cell to the neural pluripotent cell of interest.

Sendai and episomal reprogramming methods face the problem of viral clearing, and the need to screen iPSCs for sporadic genomic integration, as well as, additional traces of vector, involving residual risk associated with potential false negatives throughout the screening process. Alternatively, mRNA reprogramming presents interesting speed and productivity advantages. Cell reprogramming via mRNA transfection presently represents one of the best suited, explicitly footprint-free, cost- and timeefficient methods to generate induced pluripotent stem cells (iPSCs). mRNA transfection technology utilizes mRNA rather than DNA to release reprogramming factors into somatic cells. Although the procedure presents greater complexity in comparison with episomal and Sendai virus vectors, mRNA transfection technology is significantly more efficient. Cellular Reprogramming's platform technology enables the speedy, cost-effective, footprint-free, xeno-free generation of human induced pluripotent stem cells (iPSCs) directly obtained from human fibroblasts by using a proprietary mRNA transfection methodology. Human iPSCs represent one of the most useful technologies used in disease modeling and drug discovery, while holding the promise of stem cells based precision medicine. Cellular Reprogramming derives iPSCs from human fibroblasts on a fee-for-service basis mostly for research use only purposes.

Nicotinamide (NAM) technology has also been expanded to the field of immunotherapy, becoming an important factor in the potential treatment of several types of blood cancer and solid tumors, through the use of natural killer (NK) cells. Gamida Cell developed and patented a proprietary NAM-based cell expansion technology to develop product candidates designed to address the limitations of cell reprogramming-based therapies. NAM cell expansion technology upholds gene expression, while modulating cell function. Cellular stress is significantly diminished due to the NAM biochemical properties. NAM cell expansion technology intends to be applied in the treatment of high-risk blood cancers and rare genetic diseases, as well as, solid tumors. Gamida Cell's lead clinical candidate, NiCord $\AA$, is undergoing Phase 2/3 clinical trial as an allogeneic hematopoietic stem cell transplant solution for high-risk blood cancers and bone marrow failure disorders. NiCord ${ }^{\circledR}$ comprises umbilical cord blood expanded via NAM technology to preserve the cell capability to grow and keep stem cell behavior, and immune cells to provide immune value. NiCord $\AA$ is the first bone marrow transplant product to receive Breakthrough Therapy Designation from the US Food and Drug Administration (FDA), and has also received Orphan Drug Designation in the US and EU. NAM-NK is undergoing phase 1 clinical study used in combination with antibodies to treat relapsed or refractory multiple myeloma or non-Hodgkin lymphoma.

123Genetix, an innovative non-profit organization created to empower rare disease research, introduced and supported by the Rare Funding Team, has exceled the space by launching an unsupervised deep machine learning technology, DeepNEU, built on the bases on an entirely-connected recurrent neural network architecture, coupled with a network processing layer for each input. DeepNEU becomes artificially-induced pluripotent stem cells (aiPSC) genuineness. DeepNEU allows identifying genes and molecules needed for iPSC preservation. Although still for investigational use, the unsupervised aiPSC model is capable of converging in just 18 iterations, while non-evidencing overtraining after 1000 iterations. DeepNEU comprises 3,589 gene and proteins, which represents around $10 \%$ of the human genome, along with 27,566 nonzero relationships across 


\section{Journal of Embryology \& Stem Cell Research}

the entire neural network. Every node connects at least seven inputs and seven outputs, hence resulting in a fabulous genome web. DeepNEU has been programmed to simulate aiPSC using a precise set of reprogramming factors: OCT3/4, SOX2, KLF4 and CMYC. The final goal propelling the development and validation of DeepNEU technology is deeply associated with the possibility to model rare genetic diseases looking to the best evidenced therapeutic solutions. DeepNEU enables to create disease specific aiPSCs aiming to improve disease modeling, especially rare diseases, accelerate rapid prototyping of research advances, facilitate grant application writing, and increase the efficiency of specific biomarker identification. DeepNEU can significantly empower complex learning and analysis of genes and pathways potentially involved in the regulation of embryonic and reprogrammed somatic cells across critical signaling pathways.

\section{Key Success Factors Allowing Making Stem Cells Reprogramming A Reality for Novel Therapeutics Development}

Forthcoming innovations accelerating the pace of cell reprogramming are based on collaborative environments that promote the development of innovative therapeutics by leveraging cutting-edge genome editing and stem cell technologies. Most important approaches are directed to shed light on the process and outcome of reprogramming and provide a framework applicable to diverse temporal processes in biology [7].

There is a remarkable emphasis on bioinformatics tools enabling the analysis of epigenetics-related datasets, such as those provided by DNA methylation and hydroxymethylation analysis, ChIP-seq and RNA-seq analysis, mathematical modeling techniques in epigenomics related to both health and disease status. Also of note, it is the commitment of companies and institutions to standardize and integrate processes for personalized stem cell-based drug prediction using reprogrammed stem cells. The rapid development of core imaging technology platforms that incorporate differentiation protocols to increase the success of generating viable, functional and mature cells from either patient material or existing iPSC lines, constitutes a proven example of that trend. Similarly, iPS master cell banks are setup under GMP conditions to guarantee the development of production platforms for the on-demand generation of specific stem cell therapeutics. Relevant advances have been performed by using iPSC-derived neurons to potentially build cellular models that help to determine the underlying molecular mechanisms of several neurodegenerative disorders, including Alzheimer's disease [8].

In an attempt to distinguish the key success factors for exceling the cell reprogramming space, the following trends are highlighted [1].

Focus on marketable products for disease modeling. Novel in vitro human disease models can be created thanks to the ability to generate iPSCs from patient samples. This advancement would provide novel insights on early molecular events that regulate the pathogenesis of a broad spectrum of diseases.

Focus on making stem cells reprogramming affordable and on a massive scale. The mass production of clinicalgrade iPSCs is still a critical issue. Projects, programs and initiatives, such as the iPSCs stock for regenerative medicine are predicted to make stem cell reprogramming therapies available to a larger population at affordable costs. Similarly, clinical validation will be accelerated through the higher availability of the best suited cells.

Focus on stem cells differentiation protocols. Quality control has been another hindrance in the clinical translation of reprogrammed cells. Life scientists use whole genome sequencing to evaluate each iPS cell line. However, this methodology becomes challenging when attempting to predict cancer risk based on sequence information. Therefore, in addition to best quality and highly safe iPSCs, the focus must be on improving differentiation protocols for both particular cell lineage and complex three-dimensional structures, including tissues and organs.

Focus on reprogramming efficiency. In order to make autologous therapies available for patients, both stem cells reprogramming methods and automated culture systems must become more efficient [9-11]. Clonal variations of iPSCs must be significantly minimized in order to provide higher quality cells for clinical therapies.

\section{Discussion}

Stem cell therapies are acclaimed as the next major advancements in transforming healthcare. Pharmaceutical companies are heavily investing in expanding their cellular therapy portfolio based on stem cells reprogramming, thereby exhibiting a strong evidence of understanding future of stem cells 


\section{Journal of Embryology \& Stem Cell Research}

reprogramming in the treatment of a broad spectrum of life-threatening diseases.

Stem cells reprogramming is still bright as the medicine of the future. During the last decade, the development of stem cells therapeutics, based on stem cell differentiation protocols and direct cell reprogramming techniques, have led to over 4,500 stem cell trials registered on ClinicalTrials.gov.

\section{Conclusion}

The elucidation of the molecular mechanism of reprogramming is crucial to find novel strategies that help to improve the efficiency of reprogramming methods, and most importantly, overcome the safety concerns associated with iPSC generation.

\section{Acknowledgement}

I would like to thank all contributors from industry involved with the development and delivery of this article and Frost \& Sullivan's staff from the TechVision Group.

\section{References}

1. Van Cauwenberghe C (2019) Technological Advances Enabling Stem Cells Reprogramming. Frost \& Sullivan.

2. Spyrou J, Gardner DK, Harvey AJ (2019) Metabolism Is a Key Regulator of Induced Pluripotent Stem Cell Reprogramming. Stem cells international pp: 10.

3. Dayem AA, Lee SB, Kim K, Lim KM, Jeon TI, et al. (2019) Production of Mesenchymal Stem Cells through Stem Cell Reprogramming. International journal of molecular sciences 20(8): 1922.

4. Kumar S, Espinosa EC, Leandro AC, Curran JE, Blangero J (2019) microRNA and mRNA interactions in induced pluripotent stem cell reprogramming of lymphoblastoid cell lines. Am J Stem Cells 8(2): 28-37.

5. Nishimura K, Fukuda A, Hisatake K (2019) Mechanisms of the Metabolic Shift during Somatic Cell Reprogramming. Int J Mol Sci 20(9): 2254.

6. Van Cauwenberghe C (2019) Technologies Transforming the Biomanufacturing Landscape. Integrating Technology Innovation and Business Development (TechVision). Frost \& Sullivan Research Service D8BA-01.

7. Schiebinger G, Shu J, Tabaka M, Cleary B, Subramanian V, et al. (2019) Optimal-transport analysis of single-cell gene expression identifies developmental trajectories in reprogramming. Cell 176(4): 928-943.

8. Foveau B, Correia AS, Hebert SS, Rainone S, Potvin O, et al. (2019) Stem Cell-Derived Neurons as Cellular Models of Sporadic Alzheimer's Disease. J Alzheimers Dis 67(3): 893-910.

9. Van Cauwenberghe C (2016) Regenerative Medicine The Future of Therapy (TechVision). Re-shaping Global Healthcare. Frost \& Sullivan Research Service D6EC-01.

10. Van Cauwenberghe C (2016) Regenerative Medicine: How Stem Cell Therapy Is Innovating Healthcare. Frost \& Sullivan Market Insight.

11. Van Cauwenberghe C (2015) Hematologic Stem Cell Transplantation Enhancement Aiming to Reduce Graft Versus Host Disease. Frost \& Sullivan Drug Discovery Technology TechVision Opportunity Engine. D950-00-69. 